\section{Thyroid Cancer: A Comprehensive Guide to Clinical Management}

\author{
L. Wartofsky and D. van Nostrand, Eds.
}

New York, NY: Springer, 2016, 1,049 pages, $\$ 249$

Thyroid cancer is the most common endocrine malignancy, with an incidence that has been increasing in the United States and many other countries for several decades. Although the death rate from thyroid cancer has risen, most patients with thyroid cancer have lower stages of the disease. The thyroid cancer epidemic has led to an expansion of basic and clinical research directed at improving care, and the last 10 years have seen an astonishing transformation in the epidemiology, clinical presentation, diagnostic strategies, treatment approaches, and natural history of differentiated thyroid cancer. A rapid expansion in knowledge about the molecular underpinnings of thyroid cancer has led to new diagnostic tests and imaging approaches, important clinical trials, and therapeutic advances.

This newly produced third edition of Thyroid Cancer: A Comprehensive Guide to Clinical Management (the first edition was published in 2000 and the second in 2006) is a markedly updated and expanded volume that covers all aspects of the etiology, pathogenesis, diagnosis, and initial and long-term management of all varieties of thyroid cancer. Included in this edition are updated, extensive topics related to nuclear medicine. The book is organized into 14 parts with 107 sections. Parts I and II discuss general considerations about thyroid cancer and radioiodine scanning. Parts III, IV, VIII, and IX deal with thyroid nodules, papillary carcinomas, follicular carcinomas, and variants of thyroid cancer, respectively. Parts V, VI, and VII present postoperative management, follow-up and surveillance, and treatment, respectively. Parts X, XI, and XII discuss medullary thyroid carcinoma, thyroid lymphoma, and anaplastic thyroid cancer, respectively. Finally, parts XIII and XIV deal with new frontiers, future directions, prophylaxis against radiation exposure, and the role of low-iodine diets. There is a new chapter on the role of genetics in the devel- opment of familial nonmedullary thyroid cancer, as well as one on alternative approaches to the management of thyroid cancer. The chapters on molecular changes in thyroid cancer, molecular markers, targeted therapies, and therapeutic trials of redifferentiation agents are updated, as are the sections on pediatric thyroid cancer, in which particular attention has been given to radioiodine scanning and treatment. Thyroid cancer occurring under special circumstances, including pregnancy and renal failure, is well covered. The 95 contributors provide not only the most current review of their respective areas but also their own recommendations and approaches to the management of difficult cases.

Generally, the format is the same as in the earlier editions- that of separately discussing each type of thyroid cancer: its clinical presentation, diagnosis, surgery, pathology, follow-up, treatment, and prognosis. The figures are clear, and the tables and graphs are informative. The most current reference citations possible are provided in the bibliography of each section. Appendixes A (books and manuals), B (additional sources of information), and C (examples of forms and instructions for patients tested with ${ }^{131} \mathrm{I}$ ), as well as the index, are useful.

Thyroid Cancer: A Comprehensive Guide to Clinical Management was intended to meet the need of practicing physicians for up-to-date, clinically relevant information on the diagnosis and management of thyroid cancer. Physicians treating thyroid cancer patients will find the text comprehensive, accurate, and invaluable. I highly recommend this book to any physician involved in the care of patients with thyroid cancer.

\author{
E. Edmund Kim \\ University of California at Irvine \\ 101 The City Dr. $S$. \\ Orange, $C A 92868$ \\ E-mail: edmundek@uci.edu
}

Published online Mar. 23, 2017.

DOI: $10.2967 /$ jnumed.117.193177 